\title{
Resrarch Paper \\ The Effect of NO System on Ethidium Bromide-Induced Oxidative Stress in the Hippocampal Formation of Male Rats
}

\section{Zahedeh Rhimluoye Marjani ${ }^{1}{ }^{\circ}$, Ali Reza Alihemati ${ }^{2}$, Homeira Hatami Nemati ${ }^{10}$, ${ }^{*}$ Hatam Ahmadi $^{3}$ (1)}

1. Department of Animal Biology, Faculty of Natural Sciences, Tabriz University, Tabriz, Iran.

2. Department of Histology \& Embryology, Faculty of Medicine, Tabriz University of Medical Sciences, Tabriz, Iran.

3. Department of Basic Sciences, Farhangian University, Tehran, Iran.

\begin{tabular}{|l|l|l}
\hline $\begin{array}{l}\text { Use your device to scan } \\
\text { and read the article online }\end{array}$ & $\begin{array}{l}\text { Citation Rhimluoye Marjani Z, Ali hemati AR, Hatami Nemati H, Ahmadi H. The Effect of NO System on Ethidium Bromide-Induced } \\
\text { Oxidative Stress in the Hippocampal Formation of Male Rats. The Journal of Qazvin University of Medical Sciences. 2019; 23(3):190- } \\
\text { 201. https://doi.org/10.32598/JQUMS.23.3.190 }\end{array}$ \\
dol'https://doi.org/10.32598/JQUMS.23.3.190
\end{tabular}

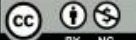

Received: 28 Feb 2019

Accepted: 11 Jun 2019

Available Online: 01 Aug 2019

Keywords:

Ethidium bromide, Nitric oxide, Oxidative stress

\section{A B STRACT}

Background Acute and chronic effects of nitric oxide on oxidative stress and neuronal demyelination have been reported in human and animal models.

Objective Oxidative stress is involved in the pathogenesis of demyelinated animals; thus, this study investigated the effects of nitric oxide system and ethidium bromide on oxidative stress in the hippocampal formation.

Methods This experimental study was performed on eight groups of male rats, as follows: control, control, ethidium bromide, L-Arginine, L-NAME, ethidium bromide + L-Arginine, ethidium bromide + L-NAME, and ethidium bromide $+\mathrm{L}$-Arginine $+\mathrm{L}-\mathrm{NAME}$. Three days after the injection of drugs into the hippocampal CA1 area, hippocampal biopsy was performed, and oxidative stress parameters were measured in this area. One-way analysis of variance (ANOVA) and posthoc tests were used for data analysis. Findings Injection of $3 \mu \mathrm{L}$ ethidium bromide into the CA1 region increased oxidative stress parameters $(P<0.01)$. Injection of $15.3 \mu \mathrm{g} / \mathrm{rat} \mathrm{L}-$ Arginine in this region stopped the response of ethidium bromide $(P<0.05, P<0.01)$; while, the injection of $15.1 \mu \mathrm{g} / \mathrm{rat} \mathrm{L}-\mathrm{NAME}$ failed to return the effects of the ethidium bromide. Moreover, the combination of $15.3 \mu \mathrm{g} / \mathrm{rat} \mathrm{L}$-Arginine and $15.1 \mu \mathrm{g} / \mathrm{rat} \mathrm{L}-\mathrm{NAME}$ only returned lipid peroxidation caused by the injection of $3 \mu \mathrm{L}$ of ethidium bromide to normal $(\mathrm{P}<0.05)$; this process failed to improve antioxidant enzymes.

Conclusion The obtained results suggested the excitatory effect of the nitric oxide system on alleviating oxidative stress induced by ethidium bromide in the CA1 region of male rats.

\section{Extended Abstract}

\section{Introduction}

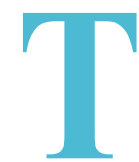

he mechanism of tissue damage in demyelinating diseases of the central nervous system is ill-defined; however, a wealth of evidence supports the role of oxidative stress and lipid peroxidation in the absence of equilibrium between the production of free radicals and intrinsic antioxidant mechanisms $[2,3]$. The antioxidant enzymes expressed in brain cells protect the neurons against reactive oxygen species and nitrogen [7]. Ethidium bromide (EB) causes the death of oligodendrocytes by producing free radicals and inducing oxidative stress, but this neurological

\section{* Corresponding Author:}

Hatam Ahmadi

Address: Department of Basic Sciences, Farhangian University, Tehran, Iran

Tel: +98 (413) 5557199

E-Mail: hahmadi@cfu.ac.ir 
damage may be restored to the physiological capacity of the central nervous system (CNS) [3].

The nitric oxide (NO) produced by nitric oxide synthesis, has beneficial effects in low amounts, but harmful effects in pathologic conditions [9]. The harmful effects of NO are due to lipid peroxidation, as well as decreasing antioxidant activity, causing oxidative stress [10]. NO level increases in the serum of patients with multiple sclerosis [12]. Previous studies have reported the antioxidant effects of NO [13].

The present study aimed to investigate the effect of injections of EB, L-arginine, and L-NAME (as well as their interaction) on the oxidative stress condition in the CA1 region of the hippocampus in male rats.

\section{Materials and Methods}

In this study, male wistar rats were used (Mean \pm SD weight: $200 \pm 50 \mathrm{~g}$ ). The drugs used in this study were EB (for demyelination), L-NAME (nitric oxide inhibitor), and L-arginine (nitric oxide stimulant). Ketamine and xylazine were used for anesthesia.

First, the animals were anesthetized by intraperitoneal injection of ketamine and xylazine. Then, the rats were placed in stereotactic devices, and the left CA1 region of the hippocampus was cannulated. During the five to seventh days of post-surgical recovery, the rats were ready for injection and test. All injections were administered on the left side of the hippocampus CA1 region.

Eight experimental groups of 7 male rats in each were selected: Control group, Control group, and five experimental groups that received EB, L-arginine, L-NAME, EB + L-arginine, EB + L-NAME, and the EB + L-arginine + L-NAME. Drug injection per microgram for each rat was

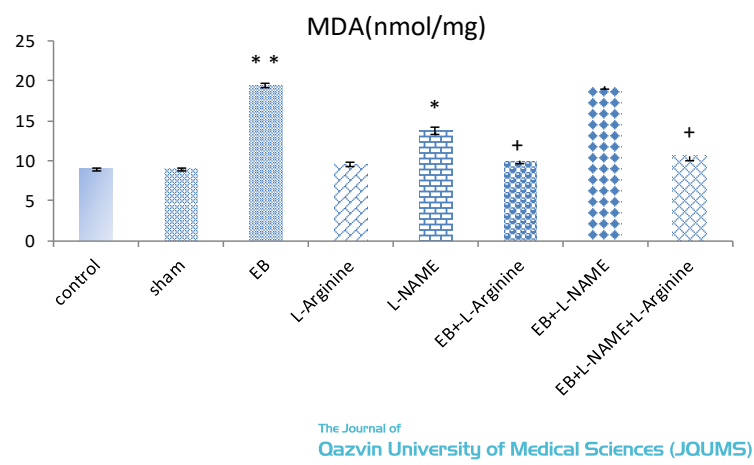

Figure 1. The effect of ethidium bromide, L-arginine and LNAME injection alone and in combination on the left side of the CA1 hippocampus on lipid peroxidation (malondialdehyde) in male rats based on previous studies $[16,17]$. The hippocampus was isolated from the brain of rats and the homogeneous tissues from the hippocampus used for biochemical analyses [13].

Lipid peroxidation by measuring the level of malondialdehyde (MDA), the activity of enzymes of glutathione peroxidase (GPX), and super oxide dismutase (SOD) in the homogenized tissue of the hippocampus was measured $[19,20]$. The one-way ANOVA test and Tukey post hoc test were used to compare the differences between the experimental groups. The significant difference was set at $\mathrm{P}<0.05$.

\section{Results}

One-way ANOVA and post hoc Tukey analysis showed that injection of $3 \mu \mathrm{L}$ of EB in the CA1 region of the hippocampus significantly increased the MDA level (Figure $1 ; \mathrm{P}<0.01$ ) and decreased the activity of GPX (Figure 2; $\mathrm{P}<0.01$ ) and SOD (Figure 3; $\mathrm{P}<0.01$ ). Applying a dose of $0.15 \mu \mathrm{g}$ L-NAME in the CA1 region of the hippocampus resulted in a significant increase in the MDA level (Figure $1 ; \mathrm{P}<0.05$ ), and a decrease in the activity of the GPX (Figure 2 ; $\mathrm{P}<0.05$ ) and SOD (Figure $3 ; \mathrm{P}<0.05$ ).

The injection of the dose of $15.3 \mu \mathrm{g}$ of L-arginine in the CA1 region of the hippocampus decreased the response of EB on the MDA (Figure 1; $\mathrm{P}<0.05$ ), GPX (Figure 2; $\mathrm{P}<0.01$ ) and SOD (Figure 3; $\mathrm{P}<0.05$ ).

The injection of the combination dose of $15.3 \mu \mathrm{g}$ of Larginine and $15.1 \mu \mathrm{g}$ of L-NAME in the CA1 region of the hippocampus reduced the response of ethidium bromide to the MDA parameter (Figure $1 ; \mathrm{P}<0.05$ ).

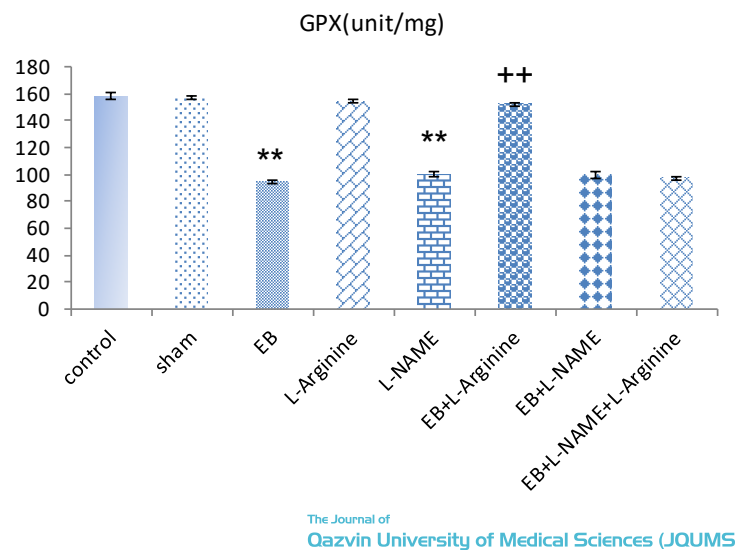

Figure 2. The effect of ethidium bromide, L-arginine and LNAME injection alone and in combination on the left side of the CA1 hippocampus on glutathione peroxidase in male rats 


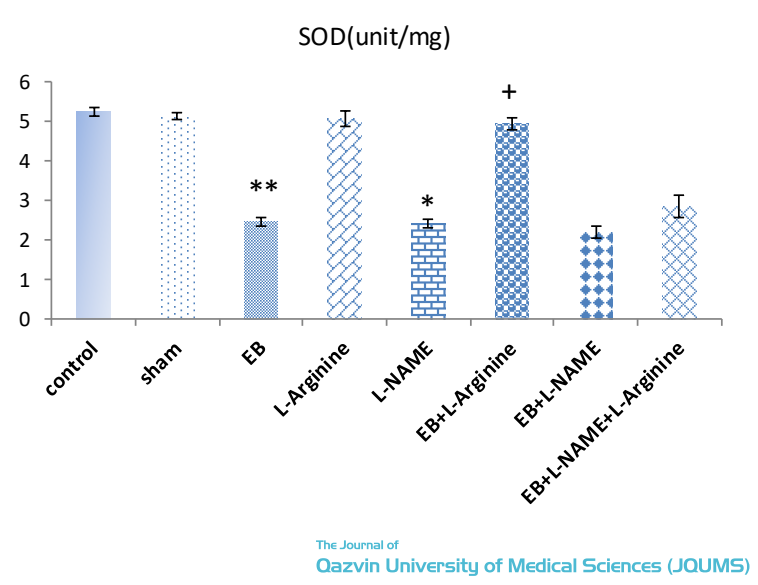

Figure 3. The effect of ethidium bromide, L-arginine and LNAME injection and their combination on the left side of the CA1 hippocampus on superoxide dismutase in male rats

\section{Conclusion}

Based on previous study, EB injection in the CA1 region of the hippocampus in male rat decreases antioxidant enzymes [21]. The study showed that injection of EB as a cause of demyelination significantly increased oxidative stress in the injection site (CA1 region of the hippocampus) in adult male rats.

The data obtained in this study showed that L-NAME (nitric oxide inhibitor) injection decreases the activity of antioxidant GPX and SOD, and increases MDA, which suggest reducing oxidative stress. Also, the use of Larginine as a stimulant for the production of $\mathrm{NO}$ in the CA1 region of the hippocampus does not affect the activity of antioxidant enzymes [7]. The findings of the study showed that the amount of these enzymes decreases in the presence of NO inhibition.

The results of this study are consistent with some previous reports, but inconsistent with some others [21, 22]. They showed that L-arginine injection stopped the response of EB to oxidative stress. Pre-treatment using NO donors can reduce the oxidative stress induced by the injection of $\mathrm{EB}$ by increasing the amount of antioxidant enzyme (GSH) [30]. Besides, the present study showed that L-NAME injection does not significantly alter the antioxidant enzymes in EB-induced demyelinated rats.

The results also indicated that co-administration of L-arginine and L-NAME in the CA1 region of the hippocampus of EB-induced demyelinated rats, although reducing the response of EB to lipid peroxidation is not capable of improving the activity of the antioxidant enzymes SOD and GSH.
Based on the results of this study, the local and acute injection of $\mathrm{NO}$ into the $\mathrm{CA} 1$ region of the hippocampus has reduced the parameters of oxidative stress (increased antioxidant status). Therefore, NO increase in this area from myelin degradation rats recovers the neurons in the region.

\section{Ethical Considerations}

\section{Compliance with ethical guidelines}

All ethical standards for working with laboratory animals have been observed.

\section{Funding}

The present paper is derived from the MSc. thesis of the first author, Zahedeh Rhimluoye Marjani, in the Animal Biology Department of Tabriz University.

\section{Authors' contributions}

Conceptualization, methodology, and supervision: Alireza Alihemati, Homeira Hatami Nemati; Research: Zahedeh Rahimluoye Marjani; Drafting: Hatam Ahmadi.

\section{Conflicts of interest}

The authors declared no conflict of interest.

\section{Acknowledgements}

The authors appreciate the close cooperation of the Research Assistant of Tabriz University for providing the funding. 


\title{
بررسى اثر سيستم نيتريكاكسايد بر استرس اكسيداتيو ناشى از اتيديوم برومايد در تشكيلات

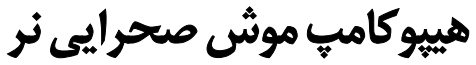

\author{
زاهده رحيملوى مرجانى' ه. على رضا على همتى'، حميرا حاتمى نعمتى'ه ، "خاتم احمدى" \\ 1- أكروه زيست جانورى، دانشكده علوم طبيعى، دانشكاه تبريز، تبريزء ايران.

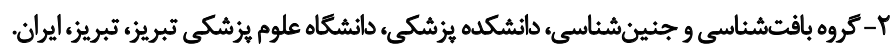 \\ r- بكروه علوم يايه، دانشكاه فرهنكيان، تهران، ايران.
}

مينه نقش حاد و مزمن نيتريكاكسايد بر استرس اكسيداتيو و موارد دميليناسيون نورونى در انسان و مدلهاى حيوانى كزارش

شده است.

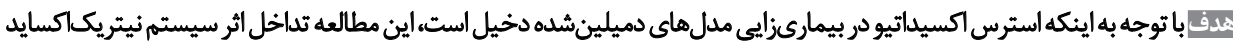

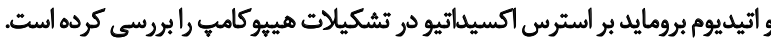

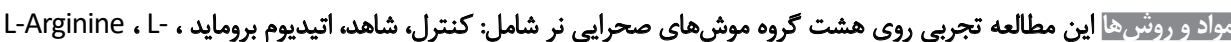

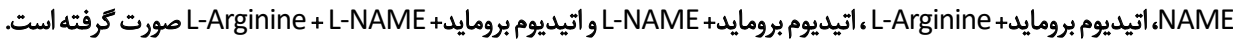

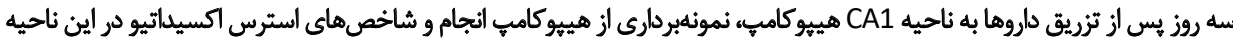

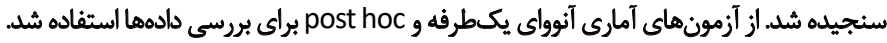

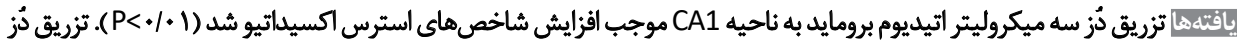

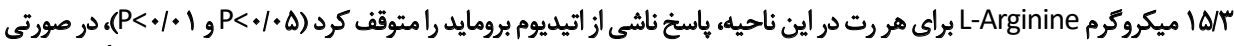

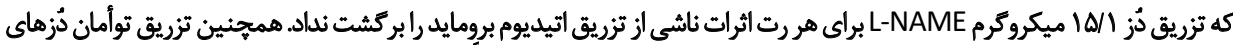

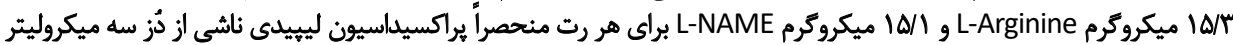

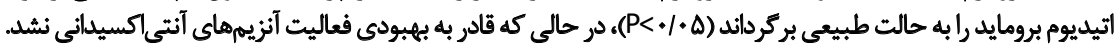

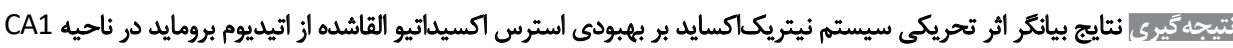

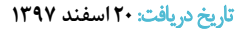

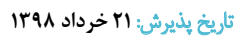

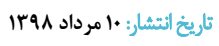

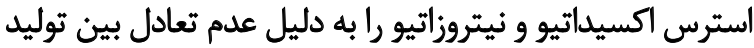

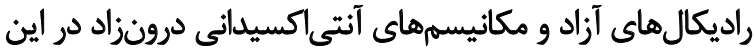

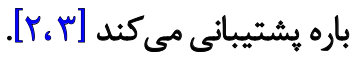

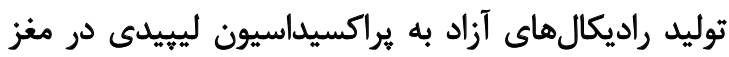

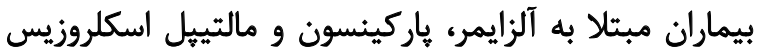

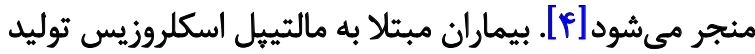

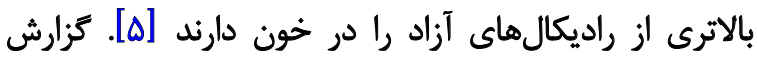

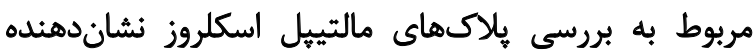

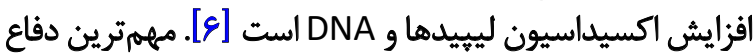

مالتييل اسكلروزيس' بيمارى خودايمنى است كه در آن ميلين

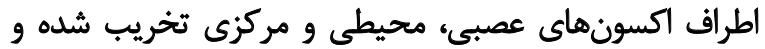

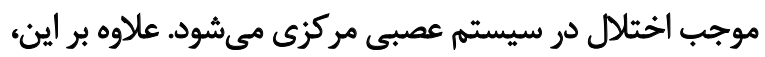

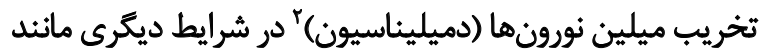

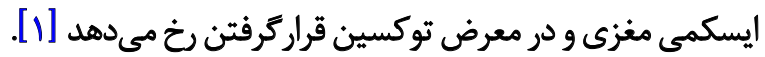

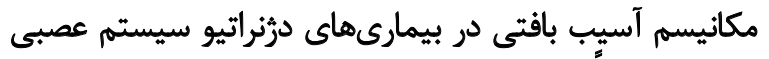

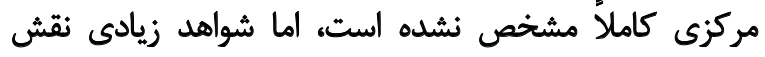

1. Multiple Sclerosis

2. Demyelination
كليدوأثهها:

اتيديوم برومايد، نيتريكاكسايد، استرس الكسيداثيو 


\section{مواد و روشها}

در اين مطالعه نجربى از وها موش صحرايى نر نرواد ويستار

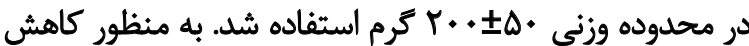

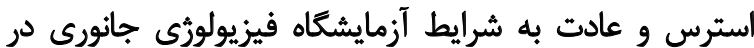

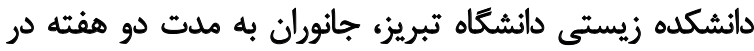

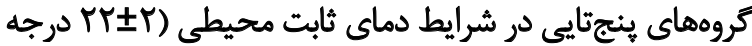
سانتى

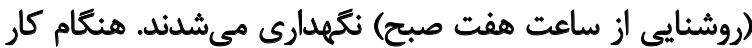

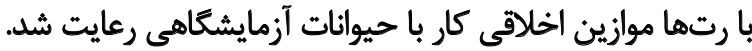

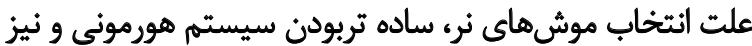

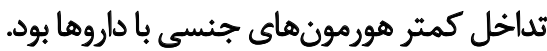

داروهاى موردبررسى در اين يُوهش عبارت بوديند ازئ اتيديوم

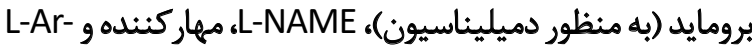

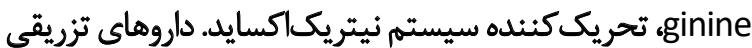

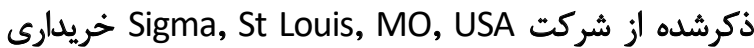

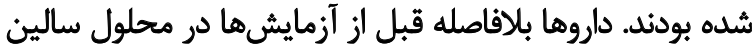

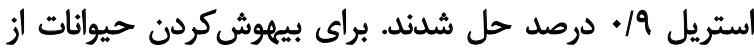

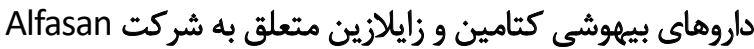

Chemical Co, Woerden, and Holland

جانوران ابتدا با تزريق درونصفاقى / / ميلىليتر از مايع

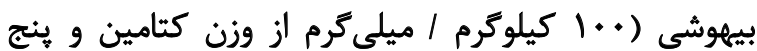

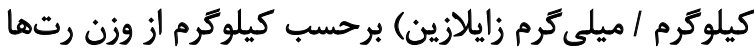

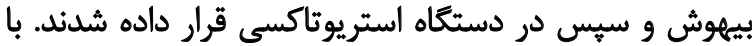

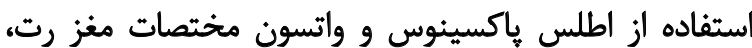

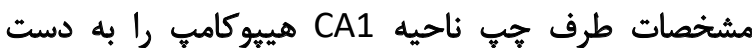

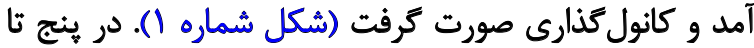

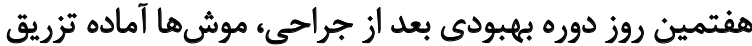

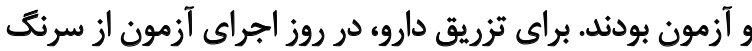

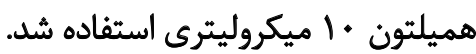

حجم تزريق براي تمام داروها در هر نوبت سه ميكروليتر به

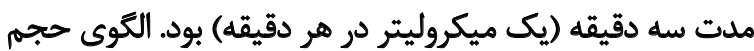

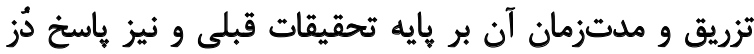

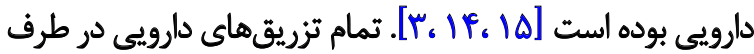

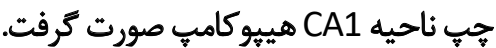

در اين مطالعه كروههاى آزمايشى هفت تايى به شرحى كه در در

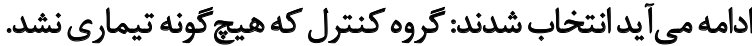

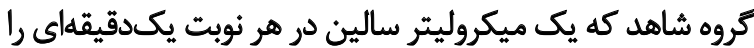

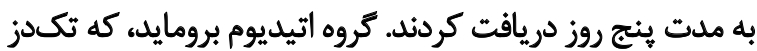

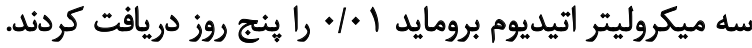

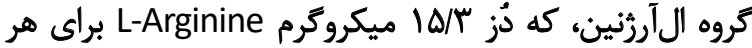

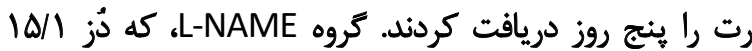

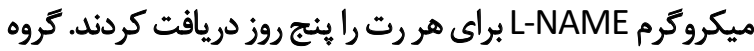

آنتىاكسيدانى با سويراكسيد دسموتاز"، كاتالاز و كلوتاتيون

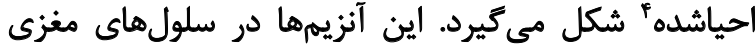

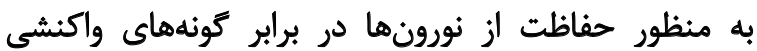

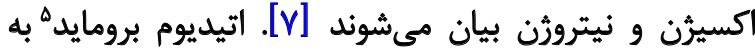

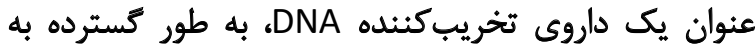

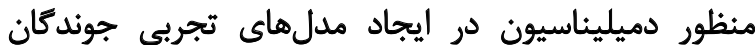

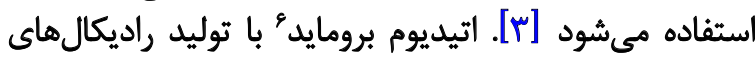

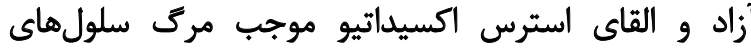

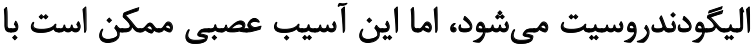

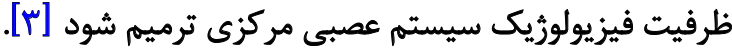
نيتريكاكسايد لمولكولي كازي است كه عمدثاً توسط آنزيم

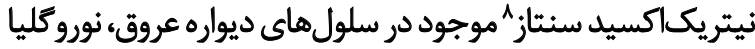

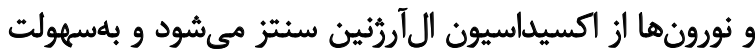

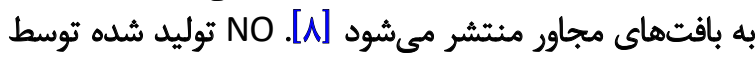

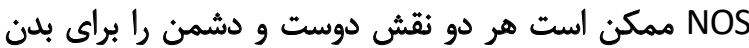

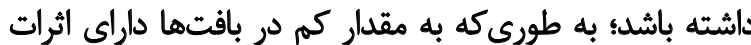

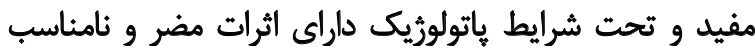

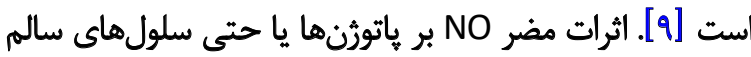

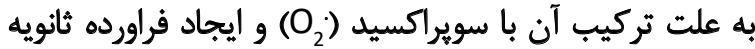

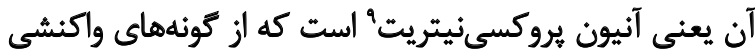

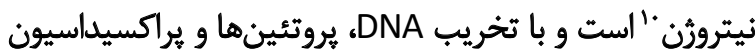

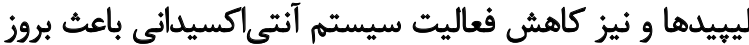

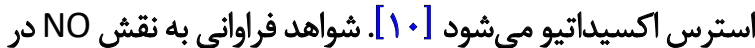

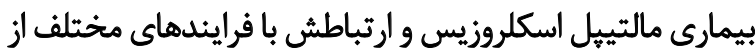

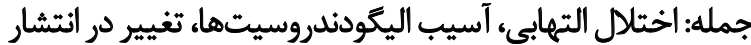

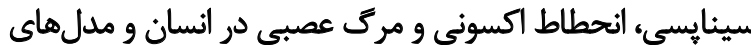

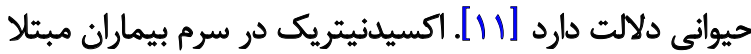

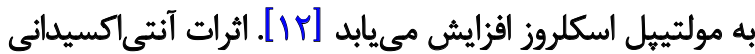

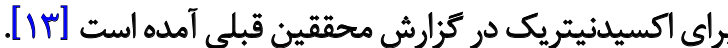
با توجه به تغيير در شاخصهاى آنتىاكسيدانى و بروز استرس

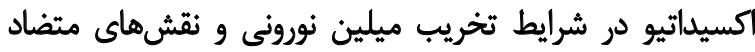

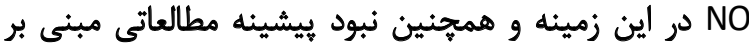

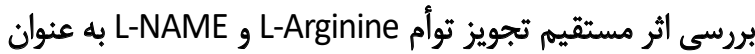

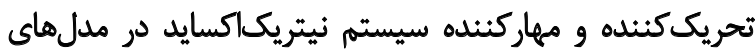

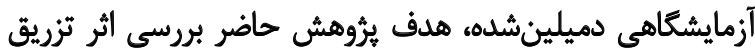

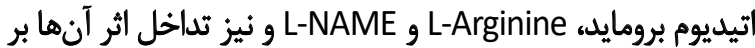
وضعيت استرس اكسيداتيو در ناحيه CA هييوكامبِ رتهائ نر است.

\footnotetext{
3. Super oxide dismutase (SOD)

4. Reduced glutathione (GSH)

5. Ethidium bromide

6. EB

7.Nitric oxide (NO)

8. NOS

9. Peroxynitrite

10. Reactive nitrogen species (RNS)
} 
هاه هانومتر از طريق طيفسنجى محلول رويى اندازهيرى

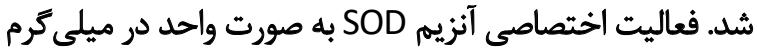

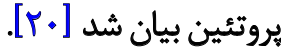

سنجش مقدار MDA و ميزان فعاليت آنزيمهاي آنتىاكسيدانى

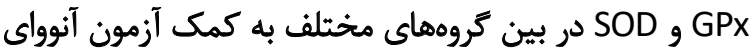

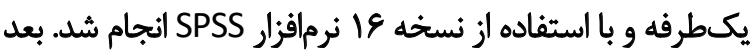

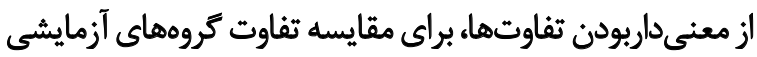

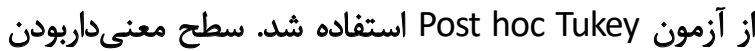

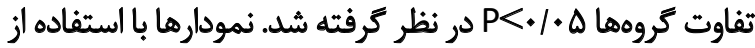

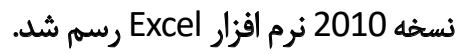

يافتهها

تزريق دُ سه ميكروليتر اتيديوم برومايد در ناحيه افريه

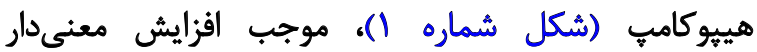

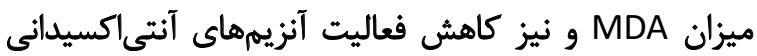

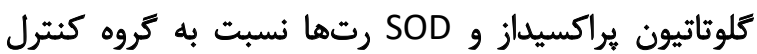

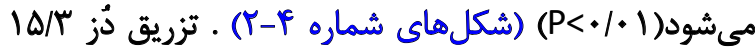

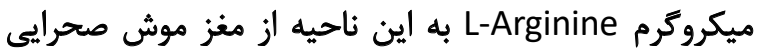

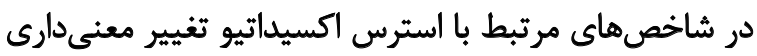

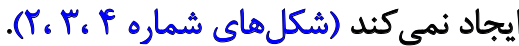

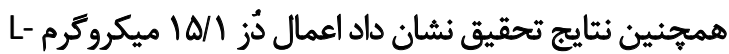

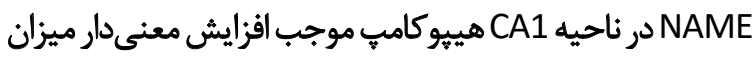

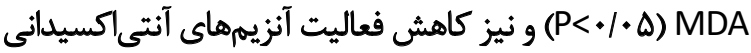

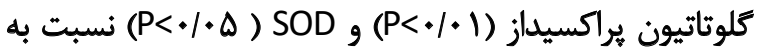

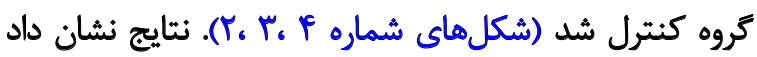

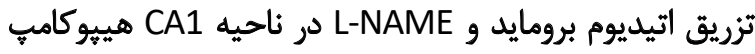

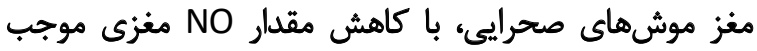

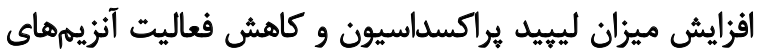

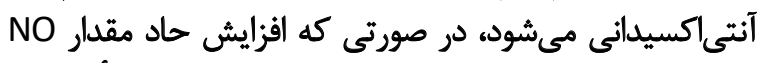

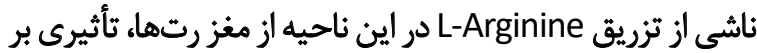
شاخصهاى مرتبط با استرس اكسيداثيو ندارد.

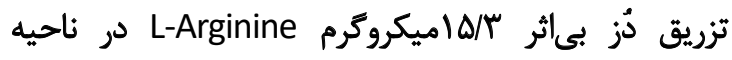

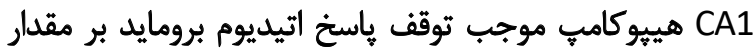

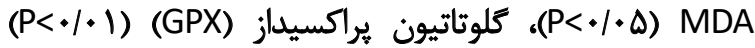

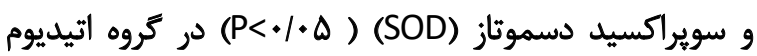

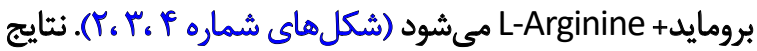

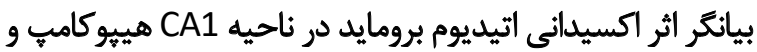

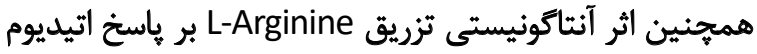

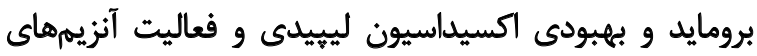
آنتىاكسيدانى در اين ناحيه از مغز است.

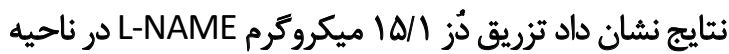

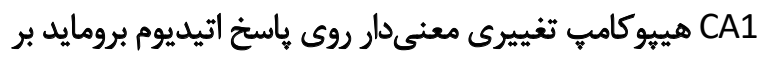

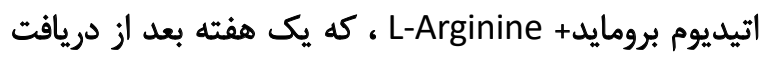

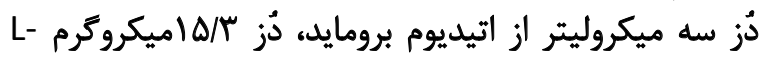

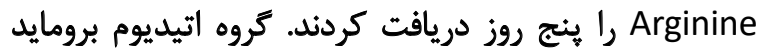

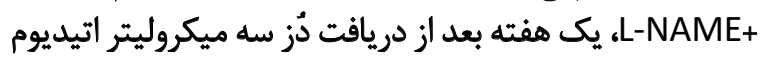

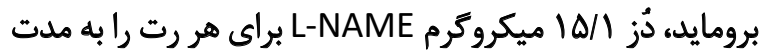

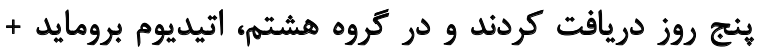

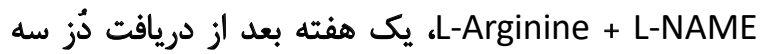

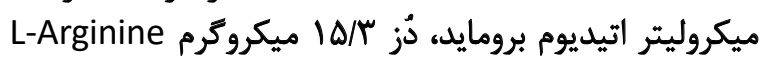

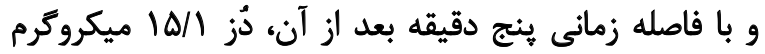

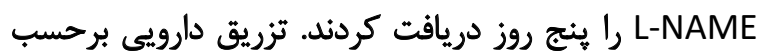

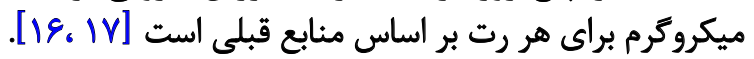
سيس موشها با ينبه آغشته به إتر، بيهوش و سر حيوان

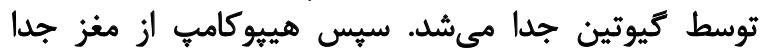

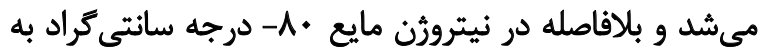

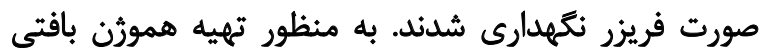

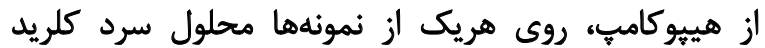

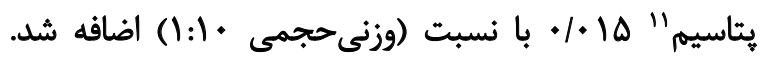

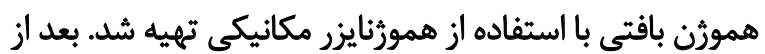

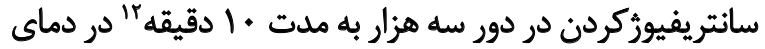
جهار درجه سانتى

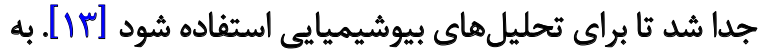

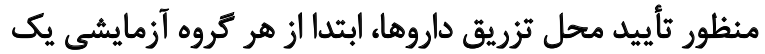

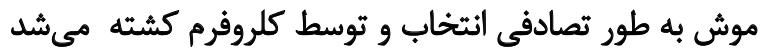

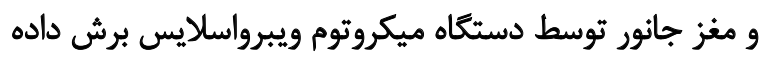

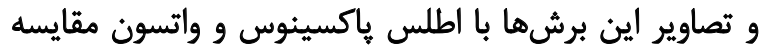

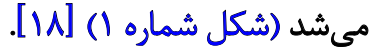

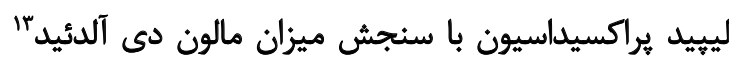

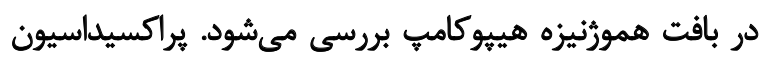

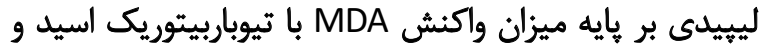

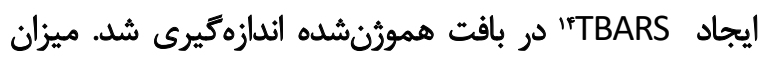

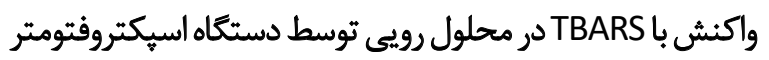

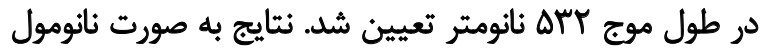

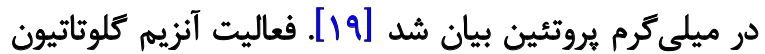

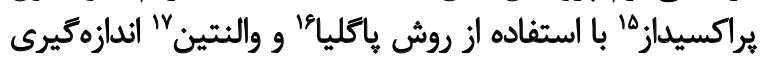

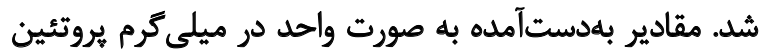

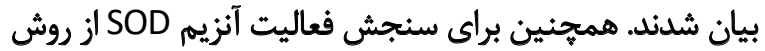

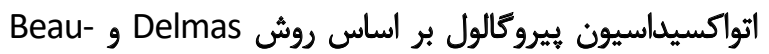

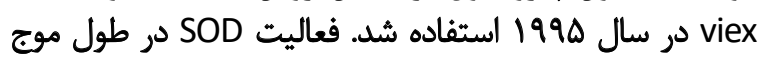

11. Potassium chloride

12. Revolution per minute

13. Malondialdehyde (MDA)

14. Thiobarbituric acid reactive substance

15. Glutathione peroxidase

16. Paglia

17. Valentine 


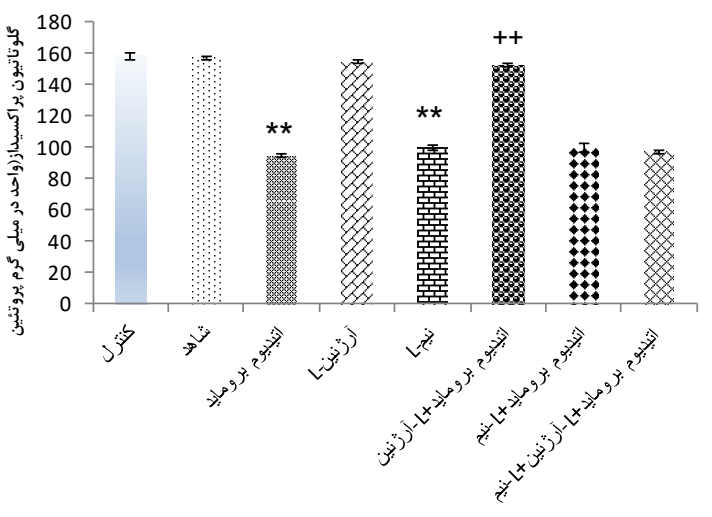

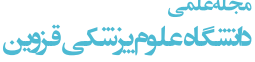

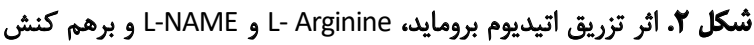

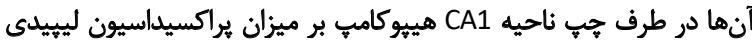
در موش صحرايى نر (MDA)

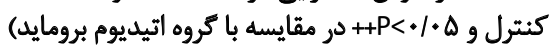

ناحيه CA1 هييوكامٍ مغز رتها موجب يراكسيداسيون

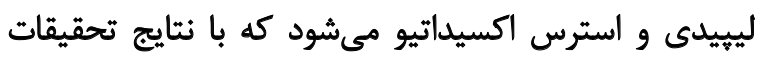

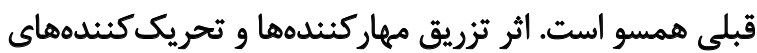

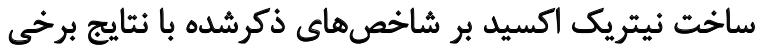

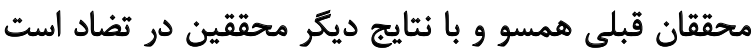

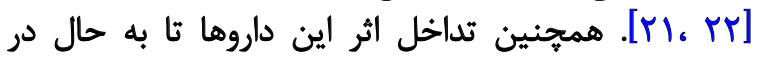
تحقيقات قبلى كار نشده است.

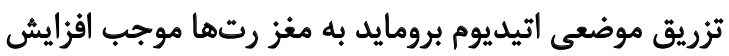

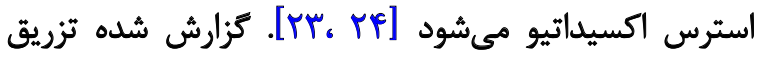

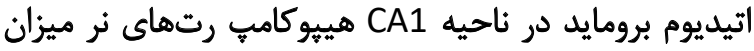

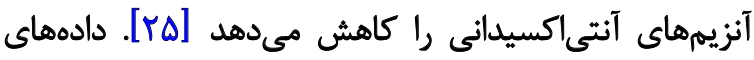

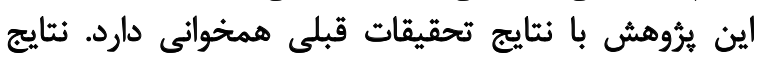

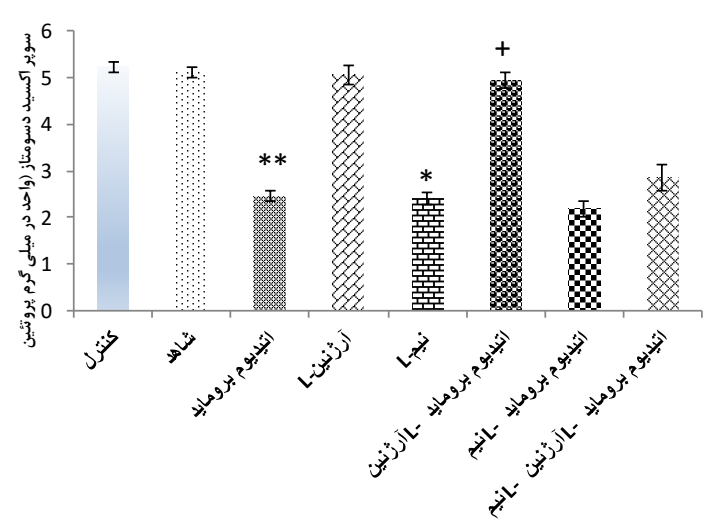

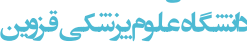

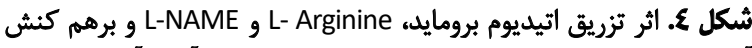

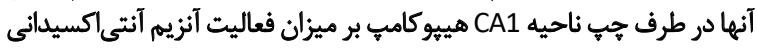

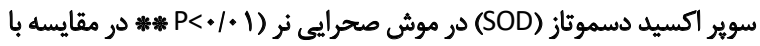

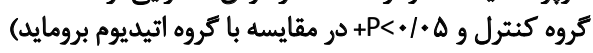

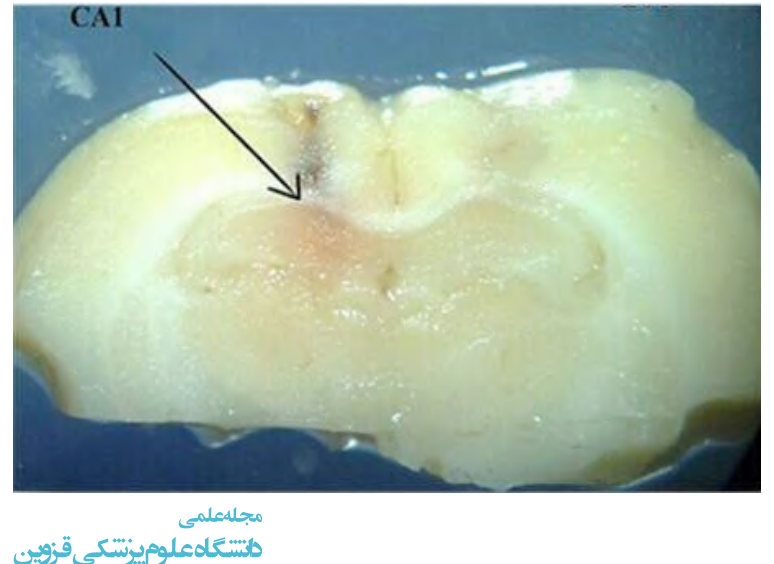

شكل ا. جايكاه تقريبي محل كائول واردشده به طرف جِي ناحيه CA1 هيبوكامب نايب

هيجكدام از شاخصهاى MDA، SOD و كلوتاتيون ثراكسيداز

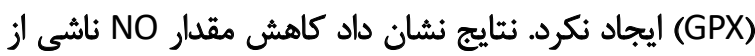

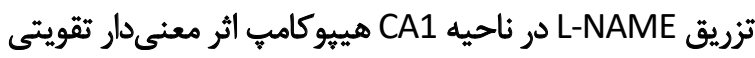

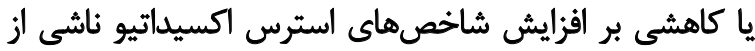
تزريق اتيديوم برومايد در اين ناحيه ندارد.

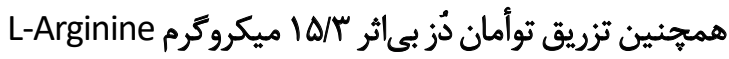

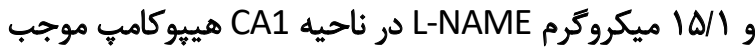

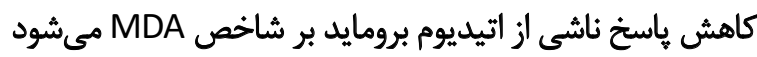

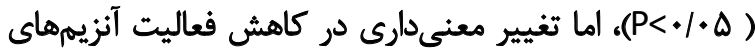

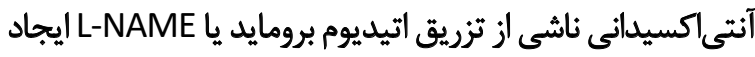

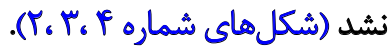

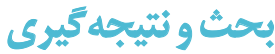

نتايج ايروهش حاضر نشان داد تزريق اتيديوم برومايد در

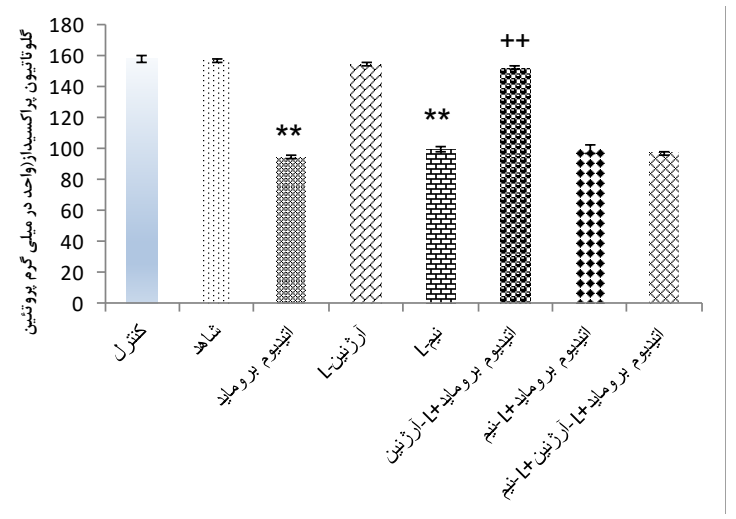

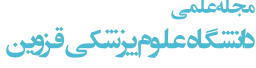

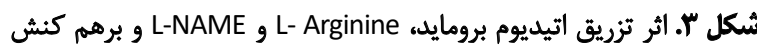

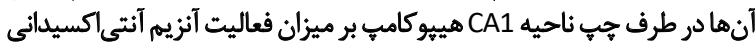

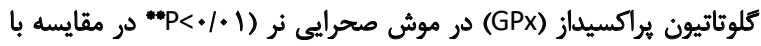

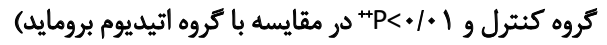


مدل هاى دميليناسيون ناشى از تزريق سم اتيديوم برومايد در مغز

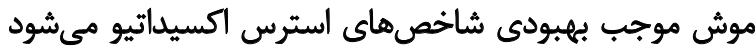

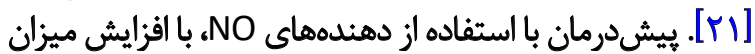

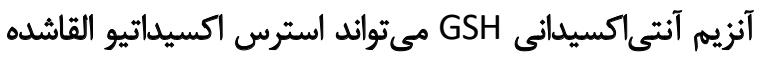

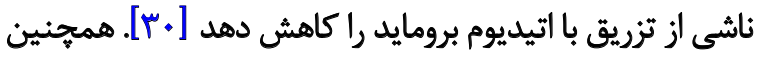

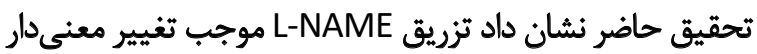

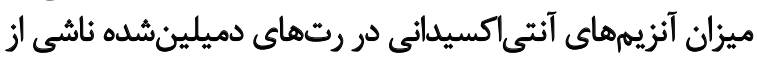

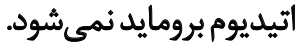

نتايج حاصل از يُوهش حاضر از تداخل اثر داروها نشان

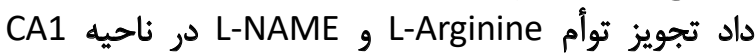

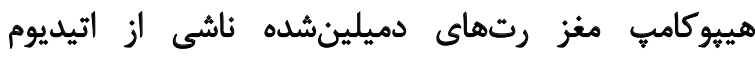

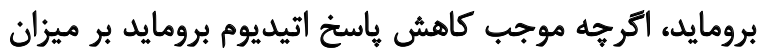

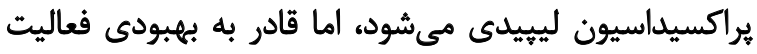

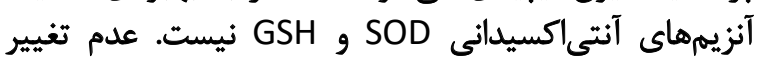

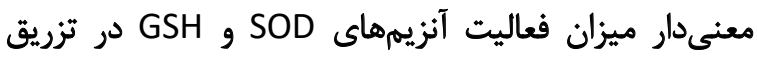

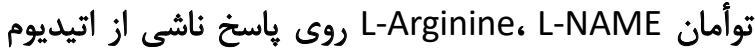

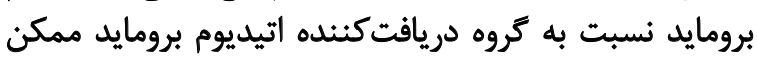

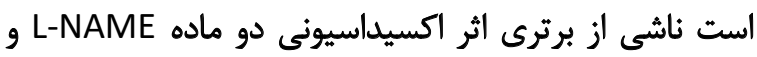

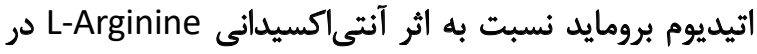

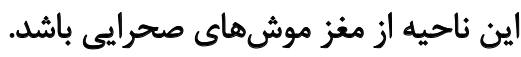

بر اساس نتايج حاصل از اين يرؤوهش (تزريق موضعى و

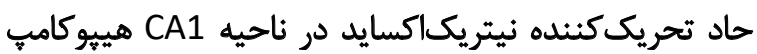

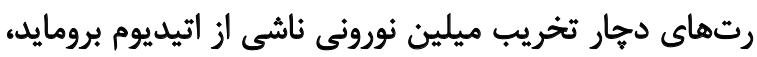

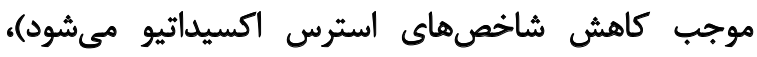

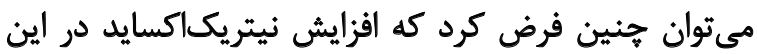

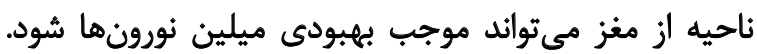

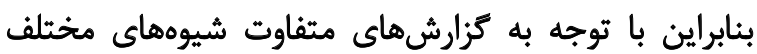

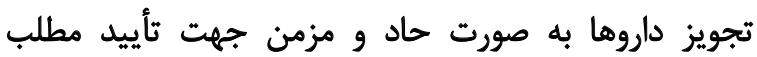

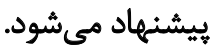
مالاحظاث اخالاقي

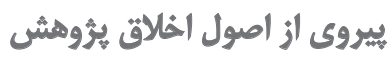
كليه موازين اخلاقي كار با حيوانات آزمايشكاهي در اين مطالعه رعايت شده است.

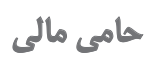

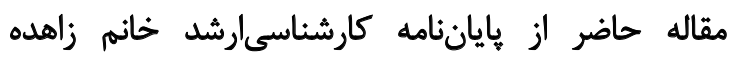

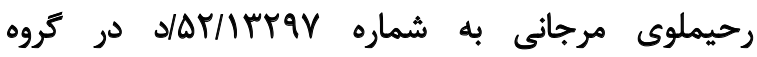
زيستشناسى جانورى دانشكاه تبريز استخراج شده است.
بلهدست آمده از مطالعه حاضر نشان داد تزريق اتيديوم برومايد

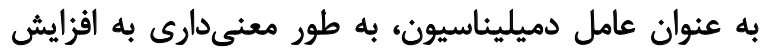
استرس اكسيداتيو در محل تزريق (ناحيه CA1 هييوكامين)

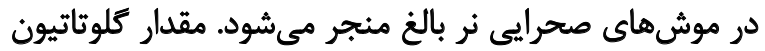

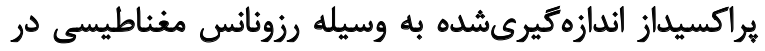

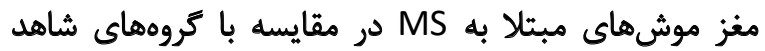

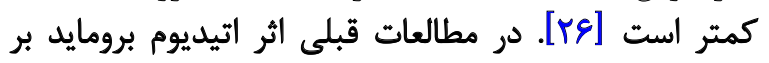

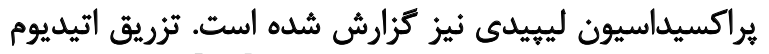

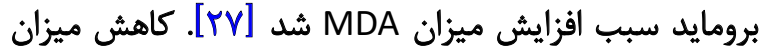

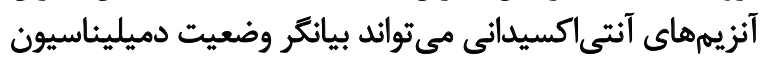

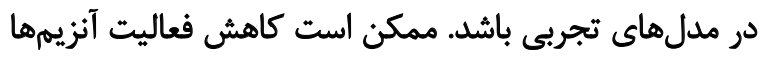

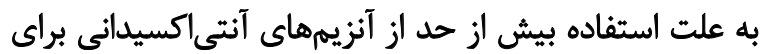

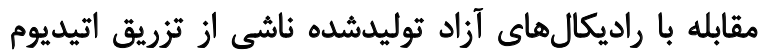
برومايد باشد [اديكالهائ

نيتريكاكسيد به عنوان يك عامل آنتىاكسيدانى بالقوه

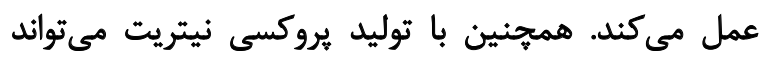

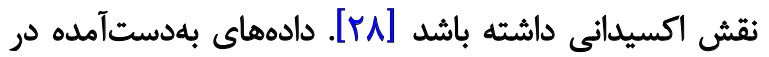

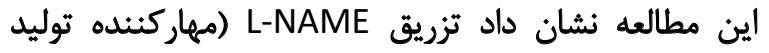
نيتريكاكسايد) موجب كاهش فعاليت آنزيمهاى آنتى اكسيداني

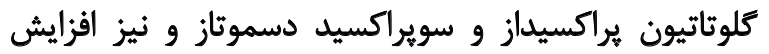

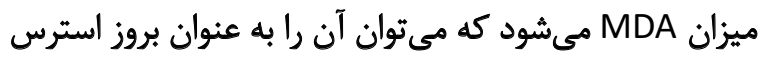

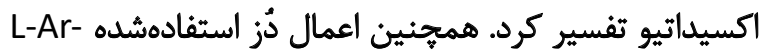

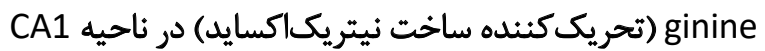

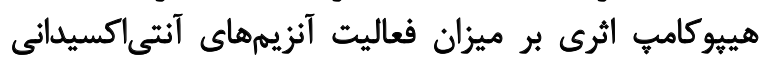

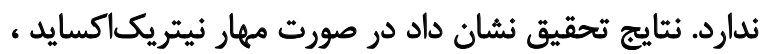

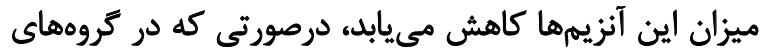

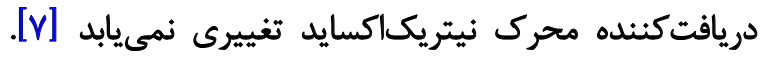

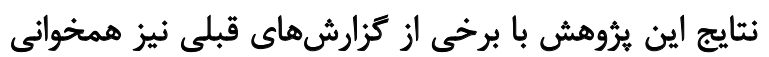

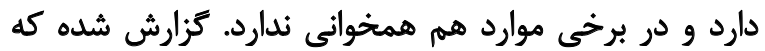

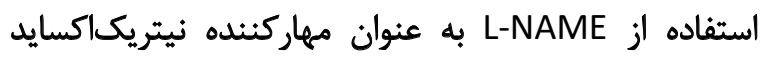

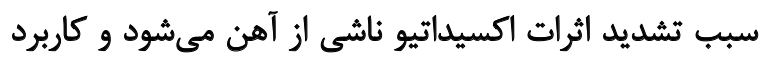
L-Arginine

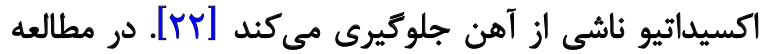

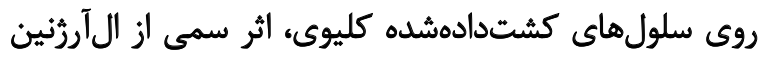

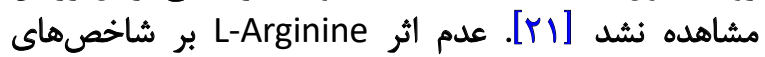

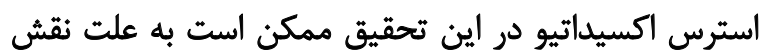
فيزيولوريك ضعيف اين ماده براى توليد NO مر در ناحيه

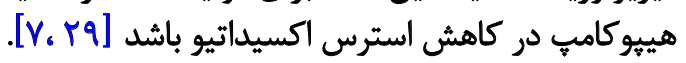

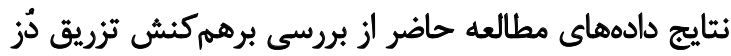

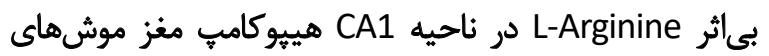

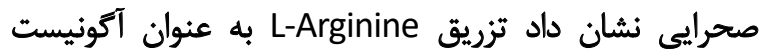

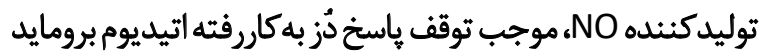

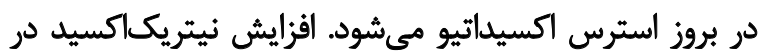




$$
\text { مشاركت نويسند متان }
$$

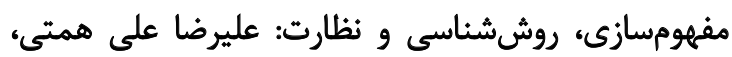

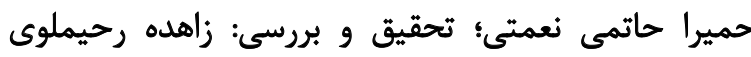

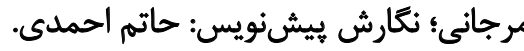

$$
\text { ت تعارض مئاقع }
$$

بنابر اظهار نويسندكان، اين مقاله تعارض منافع ندارد.

$$
\text { ساسئر: }
$$

از معاونت محترم برؤوهشى دانشكاه تبريز بابت تأمين اعتبار لازم قدردانى مى معود. 


\section{References}

[1] Lassmann H. Classification of demyelinating diseases at the interface between etiology and pathogenesis. Curr Opin Neurol. 2001; 14(3):253-8. [DOI:10.1097/00019052-20010600000001]

[2] Gilgun-Sherki Y, Melamed E, Offen D. The role of oxidative stress in the pathogenesis of multiple sclerosis: The need for effective antioxidant therapy. J Neurol. 2004; 251(3):261-8. [DOI:10.1007/s00415-004-0348-9] [PMID]

[3] Abdel-Salam OM, Khadrawy YA, Mohammed NA. Neuroprotective effect of nitric oxide donor isosorbide-dinitrate against oxidative stress induced by ethidium bromide in rat brain. Excll J. 2012; 11: 125-41. [PMID] [PMCID]

[4] Lovell MA, Ehmann WD, Butler SM, Markesbery WR. Elevated thiobarbituric acid-reactive substances and antioxidant enzyme activity in the brain in Alzheimer's disease. Neurol. 1995; 45(8):1594-601. [DOI:10.1212/WNL.45.8.1594] [PMID]

[5] Glabinski A, Tawsek NS, Bartosz G. Increased generation of superoxide radicals in the blood of MS patients. Acta Neurol Scand. 1993; 88(3):174-7. [DOI:10.1111/j.1600-0404.1993. tb04212.x] [PMID]

[6] Haider L, Fischer MT, Frischer JM, Bauer J, Höftberger R, Botond $\mathrm{G}$, et al. Oxidative damage in multiple sclerosis lesions. Brain. 2011; 134(7):1914-24. [DOI:10.1093/brain/awr128] [PMID] [PMCID]

[7] Picón-Pagès P, Garcia-Buendia J, Francisco JM. Functions and dysfunctions of nitric oxide in brain. Biochim Biophys Acta Mol Basis Dis. 2019; 1865(8):1949-67. [DOI:10.1016/j.bbadis.2018.11.007] [PMID]

[8] Tajes M, IIl-Raga G, Palomer E, Ramos-Fernández E, Guix FX, etal. Nitro-oxidative stress after neuronal ischemia induces protein nitrotyrosination and cell death. Oxid Med Cell Longev. 2013; 2013:826143. [DOI:10.1155/2013/826143] [PMID] [PMCID]

[9] Buskila Y, Amitai Y. Astrocytic iNOS-dependent enhancement of synaptic release in mouse neocortex. J Neurophysiol. 2010; 103(3):1322-8. [DOI:10.1152/jn.00676.2009] [PMID]

[10] Tajes M, Guivernau B, Ramos-Fernández E, Bosch-Morató M, Palomer E, Guix FX, et al. The pathophysiology of triose phosphate isomerase dysfunction in Alzheimer's disease. Histol Histopathol. 2013; 28(1):43-51. [DOI: 10.14670/HH-28.43]

[11] Bernhard B, Andreas von K, Katrin B. Sandau Nitric oxide and its role in apoptosis. Eur J Pharmacol. 1998; 351(3):261-72. [DOI:10.1016/S0014-2999(98)00274-X]

[12] Ibragic S, Sofic E, Suljic E, Avdagic N, Ba-jraktarevic A, Tahirovic $I$. Serum nitric oxide concentrations in patients with multiple sclerosis and patients with epilepsy. J Neural Transm. 2012; 119(1):7-11. [DOI:10.1007/s00702-011-0686-6] [PMID]

[13] Hummel SG, Fischer AJ, Martin SM, Schafer FQ, Buetner GR. Nitric oxide as a cellular antioxidant: A little goes a long way. Free Radic Biol Med. 2006; 40(3):501-6. [DOI:10.1016/j.freeradbiomed.2005.08.047] [PMID] [PMCID]
[14] Goudarzvand M, Javan M, Mirnajafi-Zadeh J, Mozafari S, Tiraihi T. Vitamins E and D3 attenuate demyelination and potentiate remyelination processes of hippocampal formation of rats following local injection of ethidium bromide. Cell Mol Neurobiol. 2010; 30(2):289-99. [DOI:10.1007/s10571-0099451-x] [PMID]

[15] Anna K L, Jere HM, Scott AS. Treatment of muscle mechanoreflex dysfunction in hypertention: Effects of L-arginine dialysis in the nucleus tractus solitari. Exp Physiol. 2013; 98(9):1337-48. [DOI:10.1113/expphysiol.2012.071563] [PMID] [PMCID]

[16] Vaseghi S, Babapour V, Nasehi M, Zarrindast MR. The role of CA1 CB1 receptors on lithium-induced spatial memory impairment in rats. EXCLI J. 2018; 17:916-34. [DOI: 10.17179/ excli2018-1511]

[17] Nasehi M, Hasanvand S, Khakpai F, Zarrindast MR. The effect of CA1 dopaminergic system on amnesia induced by harmane in mice. Acta Neurologica Belgica. 2019; 119(3):369-77. [DOI:10.1007/s13760-018-0926-8] [PMID]

[18] Paxinos G, Watson C. The rat brain in stereotaxic coordinates: hard cover edition. Amsterdam: Elsevier; 2006.

[19] Kuloghi M, Ustundag B, Atmaca M, Canatan H, Tezean AE, Cinkiline N. Lipid peroxidation and antioxidant enzyme levels in patients with schizophrenia and bipolar disorder. Cell Biochem Funct. 2002; 20(2):171-5. [DOI:10.1002/cbf.940] [PMID]

[20] Paglia DE, Valentine WN. Studies on the quantitative and qualitative characterization of erythrocyte glutathione peroxidase. J Lab Clin Med 1967; 70(1):158-69. [PMID]

[21] Mitosek-Szewczyk K, Gordon-Krajcer W, Walendzik P, Stelmasiak Z. Free radical pe-roxidation products in cerebrospinal fluid and serum of patients with multiple sclerosis after glucocorticoid therapy. Folia Neuropathol. 2010; 48(2):116-22. [PMID]

[22] Abbasnejad M, Gol A, Shirpoor A, Eskandari M. Measuring of renal Vitamin $\mathrm{E}$ for the Assessment of Iron and Nitric Oxide Interaction in Rats. J Kerman Univ Med Sci. 2004; 11(1):7-13. [In Persian]

[23] Abdel-Salam, OM E, Khadrawy YA, Salem NA, Sleem AA. Oxidative stress in a model of toxic demyelination in rat brain: The effect of piracetam and vinpocetine. Neurochem Res. 2011 36(6):1062-72. [DOI:10.1007/s11064-011-0450-1][PMID]

[24] Spanevello R, Mazzanti CM, Schmatz R, Bagatini M, Stefanello $N$, Correa $M$, et al. Effect of vitamin $E$ on ectonucleotidasea ctivities in synaptosomes and platelets and parameters of oxidative stress in rats experimentally demyelinated. Brain Res Bull. 2009; 80(1):45-51. [DOI:10.1016/j.brainresbull.2009.05.015] [PMID]

[25] Fathimoghadam H, Farbod Y, Ghadiri A, Fatemi R. Moderating effects of crocin on some stress oxidative markers in rat brain following demyelination with ethidium bromide. Heliyon. 2019; 5(2):e01213. [DOI:10.1016/j.heliyon.2019.e01213] [PMID] [PMCID]

[26] Choi IY, Lee SP, Denney DR, Lynch SG. Lower levels of glutathione in the brains of secondary progressive multiple sclerosis patients measured by $1 \mathrm{H}$ magnetic resonance chemical shift imaging at 3T. Mult Scler. 2011; 17(3):289-96. [DOI:10.1177/1352458510384010] [PMID] [PMCID] 
[27] Abdel-Salam OM, Khadrawy YA, Mohammed NA, Youness $E R$. The effect of gabapentin on oxidative stress in a model of toxic demyelination in rat brain. J Basic Clin Physiol Pharmacol. 2012; 23(2):61-8. [DOI:10.1515/jbcpp-2012-0004] [PMID]

[28] Achiron A, Gabbay U, Gilad R, Hassin-Baer S, Barak Y, Gornish $M$, et al. Intravenous immunoglobulin treatment in multiple sclerosis, effect on relapses. Neurology. 1998; 50(2):398-402. [DOI:10.1111/cns.12985] [PMID] [PMCID]

[29] Ahmadi H, Nasehi M, Rostami P, Zarrindast MR. Involvement of the nucleus accumbens shell dopaminergic system in prelimbic NMDA-induced anxiolytic-like behaviors. Neuropharmacology. 2013; 71:112-23. [DOI:10.1016/j.neuropharm.2013.03.017] [PMID]

[30] Goss SP, Hogg N, Kalyanaraman B. The effect of nitric oxide release rates on the oxidation of human low density lipoprotein. J Biol Chem. 1997; 272(34):21647-53. [DOI:10.1074/ jbc.272.34.21647] [PMID] 
This Page Intentionally Left Blank 